\section{Severe Sensory Neuronopathy in Primary Sjögren Syndrome Resulting in Charcot Arthropathy}

\section{To the Editor:}

Sjögren syndrome (SS), an autoimmune disorder affecting the exocrine glands, is known to have many extraglandular manifestations including peripheral neuropathy ${ }^{1}$. In the setting of an underlying neuropathy, progressive joint destruction is known as Charcot arthropathy.

We present a case of a 68-year-old woman with known SS presenting with functional loss of the right arm. She had presented initially 15 years earlier with keratoconjunctivitis sicca, arthralgias, and complaints of numbness and tingling of the right arm and both hands, and a positive antinuclear antibody (ANA). A diagnosis of SS was made; however, the patient was subsequently lost to followup. She was re-referred with a 6-month history of right shoulder pain. Rheumatology assessment revealed a 15-year history of right arm numbness, keratoconjunctivitis sicca, xerostomia, arthralgias, and fatigue. On history she reported an insensate right arm for "many years," as well as a recent decrease in right leg sensation. There were no additional connective tissue disease symptoms other than an intermittent facial rash. Her medical history was otherwise unremarkable and she was not taking any medications. Examination revealed edentulousness, bilateral clouded corneas, and a mild symmetric polyarthritis. The entire right arm was insensate with profound proprioceptive loss. The entire right leg had decreased sensation to light touch, pinprick, and vibration. Deep tendon reflexes were absent with no plantar reflexes. Range of motion, motor function, and gait were normal and she ambulated independently. Cranial nerve examination was unremarkable. Serology was significant for a high-titer ANA. Extractable nuclear antigens including Ro and La antibodies, dsDNA antibody, and rheumatoid factor were negative. Both the erythrocyte sedimentation rate and $\mathrm{C}$-reactive protein were elevated at $65 \mathrm{~mm} / \mathrm{h}$ and $24 \mathrm{mg} / \mathrm{l}$, respectively. Radiographic imaging of the right shoulder showed destruction of the right humeral head and glenoid, which had progressed rapidly compared to the radiographic imaging 3 months prior (Figures 1A and 1B). Magnetic resonance imaging of the cervical and thoracic spine showed no evidence of syringomyelia and no other abnormalities. Glenohumeral joint aspiration yielded no bacterial growth. Nerve conduction studies (NCS) revealed a widespread sensory neuropathy involving all 4 limbs with intact motor function and no evidence of demyelination, and was considered most likely to be neuronopathy. Electromyography was normal.

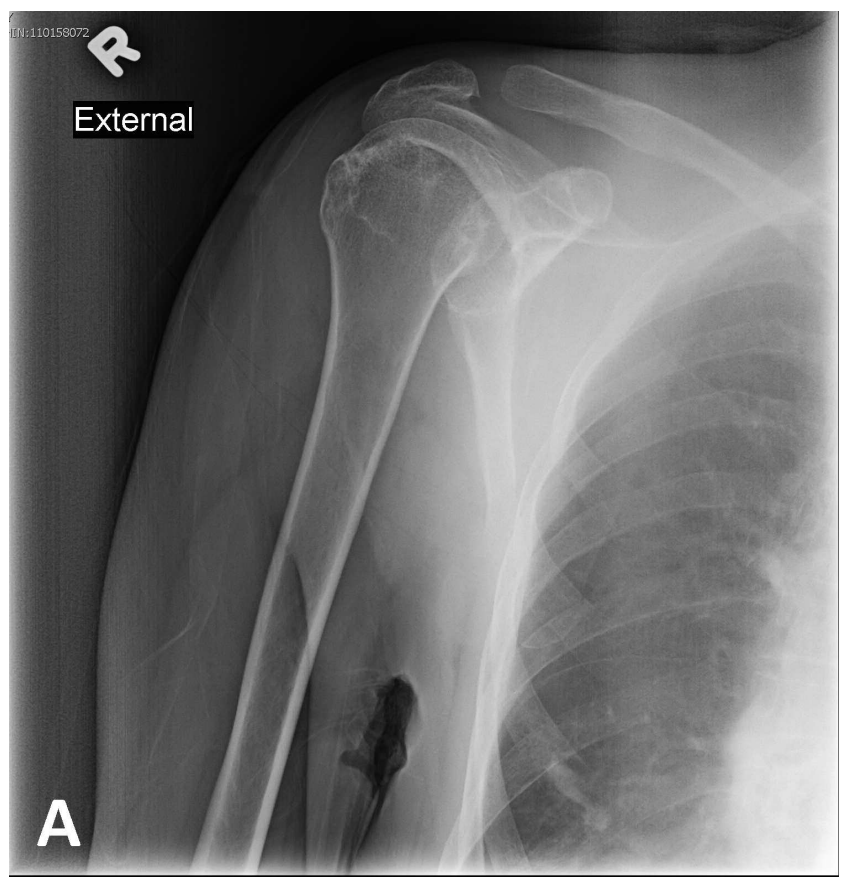

The patient was diagnosed with Charcot arthropathy of the shoulder resulting from sensory neuronopathy because of primary SS. Treatment was initiated with hydroxychloroquine, low-dose prednisone, and $2 \mathrm{~g} / \mathrm{kg}$ of monthly intravenous immunoglobulin (IVIG). She had subjective improvement in her right leg parasthesias as well as in arthralgias and fatigue, but no improvement in the right arm. Repeat NCS 1 year later revealed ongoing sensory neuropathy in both hands. After a total of 20 months of IVIG therapy, a third NCS showed ongoing sensory neuropathy with worsening of the left radial sensory response. The patient then presented with painless left foot swelling and was found to have an atraumatic, posterior calcaneal fracture suspicious for a Charcot joint about 2 years after treatment was initiated (Figure 2).

Sensory neuropathy, autonomic dysfunction, and loss of protective pain and proprioceptive sensation are thought to predispose a joint to Charcot arthropathy ${ }^{2,3}$. Charcot arthropathy is associated with diabetes mellitus, syringomyelia, spinal cord lesions, cerebral palsy, polio, leprosy, tabes dorsalis, and chronic alcoholism ${ }^{3}$. To our knowledge, no literature to date, however, has described the development of Charcot arthropathy in the setting of primary SS-associated sensory neuronopathy. Sensory neuronopathy is also called sensory ganglionopathy and ataxic sensory neuropathy. It has a prevalence of $5 \%$ to $39 \%$ of peripheral neuropathies in $\mathrm{SS}$ and its predominant feature is proprioceptive loss. It commonly presents with initial distal symptoms and a subacute or chronic onset ${ }^{4,5,6}$. There can be severe functional impairment because of the ataxia with patients becoming wheelchair- or bed-bound ${ }^{7}$.

A cohort study of 36 patients with sensory neuronopathy found that $53 \%$ and $11 \%$ have anti-Ro and anti-La antibodies, respectively ${ }^{6}$. Electrodiagnostics reveal an absence of sensory-evoked responses with intact motor responses ${ }^{4}$, consistent with our patient's findings. Nerve biopsies have shown destruction of the sensory ganglia in the dorsal root ganglia with mononuclear cell infiltration and loss of myelinated fiber density ${ }^{4,5,6}$; these findings were confirmed in an autopsy study ${ }^{7}$. Data regarding the treatment of primary SS-associated sensory neuronopathy stems from observational studies and case series. Limited responses to corticosteroids, cyclophosphamide, plasmapheresis, azathioprine, infliximab, rituximab, and D-penicillamine have been described ${ }^{1,8}$. There have also been variable responses to IVIG therapy ${ }^{6,9}$. Despite treatment, most patients had an indolent course in the longterm followup of their neurological symptoms ${ }^{9}$.

This case illustrates a severe complication of longstanding and untreated

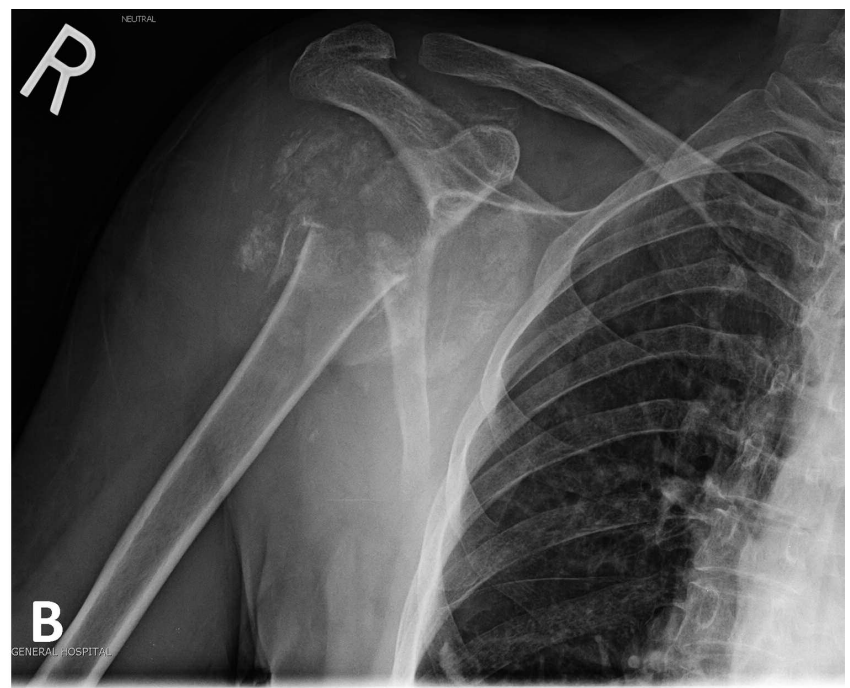

Figure 1. Right shoulder radiograph (A) 3 months prior to presentation with onset of decreased function and (B) at presentation. 


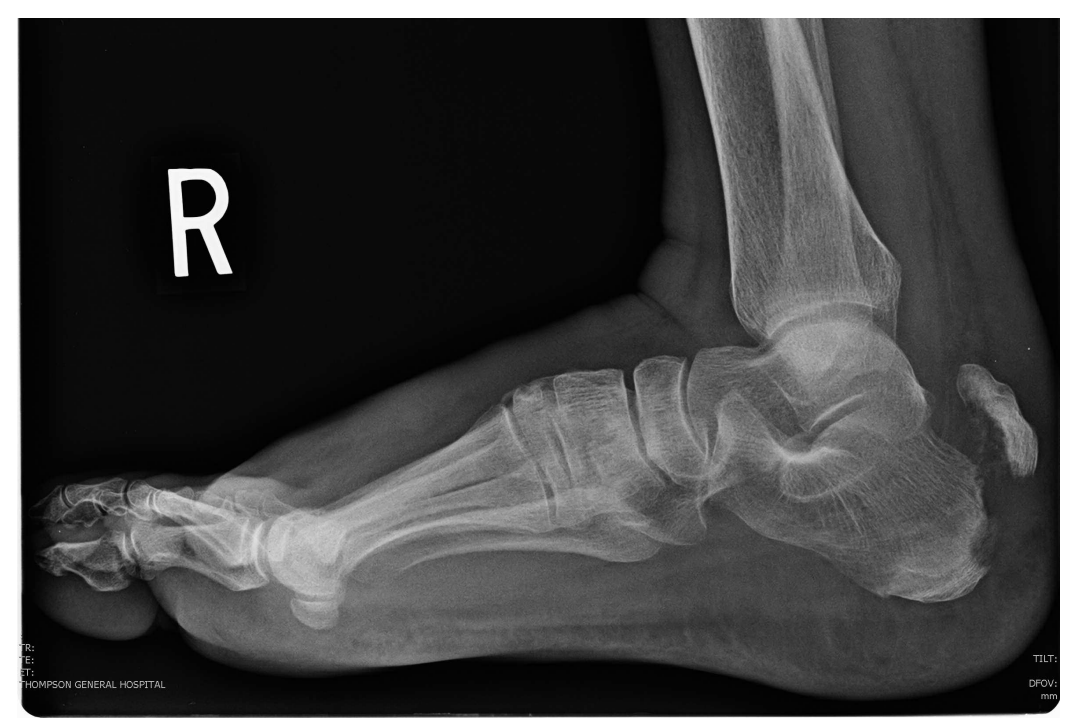

Figure 2. Right ankle radiograph with a displaced fracture of the posterior calcaneus and soft tissue swelling.

sensory neuronopathy. Though there was subjective improvement with IVIG therapy in this patient, repeat NCS showed progression of the neuronopathy and the patient developed a second Charcot joint. Monitoring of patients for the development of neurological manifestations is warranted because some patients may benefit from therapy. Further studies are necessary to determine the optimal treatment and treatment duration for patients with SS-associated sensory neuronopathies.

MAI NGUYEN, MD, Resident (PGY3) Internal Medicine, Department of Internal Medicine, Faculty of Health Sciences, University of Manitoba; CHRISTINE A. PESCHKEN, MD, MSc, FRCPC, Associate Professor, departments of Community Health Sciences and Internal Medicine, Faculty of Health Sciences, University of Manitoba, Winnipeg, Manitoba, Canada. Address correspondence to Dr. C.A. Peschken, Associate Professor, Departments of Internal Medicine and Community Health Sciences, Faculty of Health Sciences, University of Manitoba, RR149 Arthritis Centre, 800 Sherbrook St., Winnipeg, Manitoba R3A 1M4, Canada.E-mail: Christine.peschken@umanitoba.ca

\section{REFERENCES}

1. Pavlakis PP, Alexopoulos H, Kosmidis ML, Mamali I, Moutsopoulos HM, Tzioufas AG, et al. Peripheral neuropathies in Sjögren's syndrome: a critical update on clinical features and pathogenetic mechanisms. J Autoimmun 2012;39:27-33.

2. Jude EB, Boulton AJ. Update on Charcot neuroarthropathy. Curr Diab Rep 2001;1:228-32.
3. Chisholm KA, Gilchrist JM. The Charcot joint: a modern neurologic perspective. J Clin Neuromuscul Dis 2011;13:1-13.

4. Birnbaum J. Peripheral nervous system manifestations of Sjögren syndrome: clinical patterns, diagnostic paradigms, etiopathogenesis, and therapeutic strategies. Neurologist 2010;16:287-97.

5. Chai J, Logigian EL. Neurological manifestations of primary Sjogren's syndrome. Curr Opin Neurol 2010;23:509-13.

6. Mori K, Iijima M, Koike H, Hattori N, Tanaka F, Watanabe H, et al. The wide spectrum of clinical manifestations in Sjögren's syndrome-associated neuropathy. Brain 2005;128:2518-34.

7. Kawagashira Y, Koike H, Fujioka Y, Hashimoto R, Tomita M, Morozumi S, et al. Differential, size-dependent sensory neuron involvement in the painful and ataxic forms of primary Sjögren's syndrome-associated neuropathy. J Neurol Sci 2012;319:139-46

8. Font J, Ramos-Casals M, de la Red G, Pou A, Casanova A, Garcia-Carrasco M, et al. Pure sensory neuropathy in primary Sjögren's syndrome. Longterm prospective followup and review of the literature. J Rheumatol 2003;30:1552-7.

9. Takahashi Y, Takata T, Hoshino M, Sakurai M, Kanazawa I. Benefit of IVIG for long-standing ataxic sensory neuronopathy with Sjögren's syndrome. IV immunoglobulin. Neurology 2003; 60:503-5.

J Rheumatol 2016;43:7; doi:10.3899/jrheum.160137 